\title{
Die klinische Medizin und die Naturwissenschaften um 1800
}

\author{
Von Erwin H. Ackerknecht
}

Über die enormen Leistungen der Naturwissenschaften um 1800 braucht man nicht viel Worte zu verlieren. Einige Namen rufen sie auch dem Nichtspezialisten schnell ins Gedächtnis zurück. Wir nennen hier nur Lagrange, Gauß, Laplace, Lavoisier, Priestley, Galvani, Volta, Gay-Lussac, Dalton, Davy, Berzelius, Chevreuil, Spallanzani, Lamarck, Cuvier, Geoffroy St. Hilaire. Um die klinische Medizin war es hingegen nicht besonders gut bestellt, mit Ausnahme der englischen und französischen Chirurgie. JeŁzt begann aber auch die Medizin schnell aufzusteigen. Paradoxerweise vorerst nicht durch Anwendung der Naturwissenschaften. Die klinische Medizin zog sich mehr oder weniger, wie der legendäre Baron von Münchhausen, um diese Zeit, dort wo ihr dies Manöver glückte, am eigenen Zopf aus dem Sumpf heraus. Die Entwicklung war keineswegs gradlinig. Um eine nun schon über ein Vierteljahrhundert alte Periodisierung des Schreibenden zu verwenden ${ }^{1}$ : auf die «Krankenbett-» und "Bibliotheksmedizin» folgte nicht «logisch» die «Labormedizin», welche sich erst nach 1850 in Deutschland durchsetzte, sondern die sogenannte "Spitalmedizin», welche sich am besten in Frankreich entwickelte. Dies erklärt sich teilweise durch die vorschnelle Anwendung von Chemie und Physik auf die Medizin im 17. und 18. Jahrhundert (Iatrophysik und Iatrochemie), welche zu großen Enttäuschungen geführt hatte. So kam es vorübergehend zu einer Lockerung der Beziehungen zwischen Medizin und Naturwissenschaften, deren Hauptträger die Ärzte ja bis jetzt gewesen waren, wie der englische Ausdruck "physician» noch immer anzeigt. Zu einer Wiedervereinigung kam es erst in der Mitte des 19. Jahrhunderts.

Ich muß mich im folgenden auf eine Darstellung der Situation in den drei medizinisch wichtigsten Ländern Europas: Frankreich, Deutschland und Großbritannien beschränken. In Frankreich kam der medizinische Umschwung eindeutig mit der Revolution (die wissenschaftliche Aufwärtsentwicklung hatte schon vorher eingesetzt). Man muß Daremberg ${ }^{2}$ zustimmen, wenn er feststellt, daß die vorrevolutionären traditionalistischen französischen Kliniker wie Lorry, Lieutand oder Hallé nicht besonders hervorragend waren. Durch die Abschaffung der alten Fakultäten und die Eröffnung der neuen Medizinschulen durch den Konvent im Jahre 1794 wurde nun «Beobachtung» das Losungswort der Pariser Schule ${ }^{3}$. Die medizinische Lehre wurde vom Pult in die großen Spitäler und auf die Präparierböden verlegt. Wie Fourcroy, der Berichterstatter über das neue Gesetz, es ausdrückte: «peu lire, beaucoup voir, beaucoup faire». Der erste hervorragende Kli- 
niker der neuen Schule, Pinel, war allerdings noch in erster Linie «Nosologe» und darum stark von den taxonomischen Ideen der Zoologie und der Botanik beeinflußt. Er basierte andererseits aber bereits seine neuen Krankheitsbilder weitgehend auf die pathologische Anatomie, die nun von den Chirurgen mit Macht in die klinische Medizin hineingetragen wurde. Die zweite Säule der Pariser medizinischen Forschung war die physikalische Diagnose durch Auskultation und Perkussion, also eine aktive Form der Beobachtung. Die dritte war die Statistik. Sie ist im allgemeinen mit dem Namen von Pierre Louis verknüpft, und es wird übersehen, daß sie bereits von Pinel, Bayle, Laënnec, Esquirol, Rostan, Bouillaud, Rayer usw. praktiziert wurde. Alle drei Säulen der neuen Medizin konnten nur im Spital errichtet werden. Es gab zwar in der neuen Schule von 1794 im Gegensatz zur alten Fakultät Lehrstühle für medizinische Chemie (kombiniert mit Pharmakologie), medizinische Physik (kombiniert mit Hygiene) und Naturgeschichte. Sie waren aber wirklich nur Hilfswissenschaften (wie Alibert ${ }^{4}$ triumphierend sagte: «nicht die Hilfswissenschaften haben uns erobert, sondern wir haben sie erobert»), und die Lehrer waren auch darnach. Es ist typisch, daß der erste Theoretiker der Schule, Cabanis, an Stahl besonders rühmt, daß er die Physik und die Chemie aus der Medizin verbannt habe, und die Entdeckung von Harvey für praktisch wertlos hält. Die tiefe Enttäuschung über die Pseudo-Ursachenforschung der Vergangenheit klingt deutlich nach, sowie der jakobinische Utilitarismus.

Die Schule verneinte die drei Säulen, auf denen die kommende Labormedizin aufgebaut werden sollte: Experimentalphysiologie und -pathologie, Mikroskopie und Biochemie. Es gab auch in Paris um 1800 geniale Experimentalpathologen und -physiologen wie Legallois und den Laplace nahestehenden Magendie; aber sie blieben ohne Einfluß auf die Klinik. Pierre Louis lehnte das Experiment ab, wie schon Fodéré, Chaussier, Royer-Collard, Gall und Georget. Es gab in Paris geniale Biochemiker wie die Entdecker der Alkaloide; aber Corvisart verweigerte zum Beispiel die Einrichtung von Labors in den Kliniken, wie sie Fourcroy vorschlug. Bichat, der jung verstorbene Abgott der jungen Schule, fand sowohl Physik wie Chemie überflüssig. Er war ja, wie so viele andere, Vitalist. (Er kultivierte aber das Tierexperiment.) Es gab auch gute französische Mikroskopiker. Aber das Mikroskop wurde sowohl von Bichat wie von Laënnec, Cabanis und Trousseau abgelehnt. Kein Wunder, daß Oliver Wendell Holmes als amerikanischer Student in Paris den Eindruck hatte, die Wissenschaften würden nur aus mnemotechnischen Gründen den Medizinstudenten gelehrt.

Bei den meisten führenden Vertretern der Schule hatte sich ein empirischer Wissenschaftsbegriff gebildet: Wissenschaft ist Tatsachen (Laënnec). Dieser Wissenschaftsbegriff wurde kombiniert mit einem Kausalagnostizismus. Es gab Aus- 
nahmen von dieser ablehnenden Haltung gegenüber den Grundwissenschaften, wie Dupuytren und Bretonneau; am Ende der Glanzzeit der Pariser Schule wurden sie teilweise von einigen wie Andral, Pierre Louis oder Gavarret integriert; aber der typische Vertreter der Schule war doch Trousseau, welcher feststellte, daß Wissenschaft denkfaul mache und der Kliniker mehr «Kunst» als Wissenschaft brauche ${ }^{5}$. Dieses Kunstgerede, welches sich immer einstellt, wenn Unwissenheit bemäntelt werden soll, finden wir bei fast allen Pariser Lehrern von Cabanis bis Trousseau. Trotzdem hat diese Schule Gewaltiges geleistet. Unsere Auffassungen von Tuberkulose, Unterleibstyphus, Diphtherie sind von ihr geprägt. Sie hat das Gebiet der Herzkrankheiten, Lungenkrankheiten, Hirnkrankheiten, Geschlechtskrankheiten, Kinderkrankheiten, Geisteskrankheiten neu erschlossen. Aber sie geriet in eine Sackgasse und begann zu stagnieren. Es ist typisch, daß die Franzosen weder die Anästhesie noch die Asepsis entdeckt haben, denn dazu hätte man Chemie und Mikroskopie treiben müssen. Die Franzosen haben zwar das Gebiet der mikroskopischen Krankheitserreger weitgehend erschlossen, aber durch zwei Chemiker, Raspail und Pasteur!

Wenden wir uns nun Deutschland zu. Während man in Frankreich offen gegen die «Hilfswissenschaften» sprach, leistete man andererseits in der Klinik Großes. In Deutschland redete man überhaupt nur von Wissenschaft und leistete in der Klinik so gut wie gar nichts. Die Wissenschaft war nämlich gar keine (wie heute der Dialmat oder ein großer Teil der Tiefenpsychologie, die sich ja auch so gern als Wissenschaft bezeichnen), sondern Philosophie, in diesem Fall Schellingsche Naturphilosophie. Um den halbspekulativen Systemen des 18. Jahrhunderts (Hoffmann, Stahl, Boerhaave) zu entgehen, stürzten sich die Deutschen paradoxerweise unter dem Einfluß einer außerordentlich kraftvollen Persönlichkeit, Schellings, in die reine Spekulation. Für Schelling war die Arzneiwissenschaft die Krone aller Wissenschaften. Wissenschaft war aber gleich Naturphilosophie ${ }^{6}$. Sie bestand aus einer Reihe von «Prinzipien» (aprioristischen Sätzen), die sich selbst die Richtung gaben ${ }^{7}$. Schelling war ausdrücklich gegen das Experiment. Die Natur war ja eigentlich Geist. Einige dieser Prinzipien waren Polarität (eine falsch verstandene Entwicklung der neuen Elektrizitätslehre), Kreislauf, Steigerung, Organismus, Dynamik, Vitalismus ${ }^{8}$ vermischt mit der vom Edinburger Alkoholiker John Brown ${ }^{9}$ wieder aufgewärmten dualistischen Lehre der römischen Methodiker, in der nun Asthenie und Irritation als die zwei UniversalKrankheitsursachen bezeichnet wurden. Eine solche Bewegung war natürlich nur möglich in einem Lande, in dem schon im 18. Jahrhundert Bücher über die "philosophischen» Bandagen erschienen waren. Darum wurden ihre Vorkämpfer auch nicht nur der Philosoph Schelling, der Journalist Görres, der Dichter Novalis, 
die Provinzärzte Röschlaub und Marcus, sondern sogar ein Johann Peter und Joseph Frank. Görres fand zum Beispiel, man müsse «die Projektion des Weltbaus im Organismus» nachweisen ${ }^{10}$. Novalis ${ }^{11}$ empfahl die Benutzung des «Zauberstabs der Analogie», mit dem der nun wiederentdeckte Paracelsus schon so Großes geleistet hatte wie die Erklärung des Nordlichts als Menstruation der Erde oder der Epilepsie als Erdbeben des Körpers. Zum Schluß landeten die meisten Romantiker nicht zufällig beim Katholizismus und der Sünde als Krankheitsursache. Sie hatten auch geistreiche Ideen von Krankheit als «Regression». Für Karl Richard Hoffmann war die Skrofulose zum Beispiel ein Zurücksinken in das Insektenstadium. Karl Wilhelm Stark unterschied zwischen höheren und niedereren Krankheiten und betrachtete die Krankheit als "Parasiten » ${ }^{12}$. Die romantische Medizin ergriff die deutschen Ärzte wie ein Rausch. Manche, die vorher recht nüchtern waren, wie Reil oder die Franks, schlossen sich ihr an. Andere lebten und starben in ihr. Überwunden wurde sie durch Männer wie Johannes Müller und Lukas Schönlein, die ihr in ihrer Jugend selbst gehuldigt hatten. Neben den Romantikern gab es auch in Deutschland immerhin ein paar tüchtige Empiriker, wie Peter Krukenberg in Halle oder Moritz Romberg in Berlin ${ }^{13}$. Sehr verbreitet war auch das Geschlecht der Eklektiker, für das Hufeland typisch ist ${ }^{14}$. Die besten Vertreter der deutschen Medizin waren zweifellos die (in Paris ausgebildeten) Chirurgen. Wenn auch die Klinik nicht von der Romantik profitierte, so doch einige biologische Spezialitäten wie die Embryologie. Wien übersprang fast die romantische Phase, akzeptierte das Pariser Modell und wurde damit führend in der deutschen Medizin ${ }^{15}$.

In Großbritannien ist, wie so häufig, die Situation unübersichtlich. Auch dort war man von Iatrophysik und Iatrochemie enttäuscht. Die Reaktion war aber nur ein schlichter Empirismus, den die deutschen medizinischen Reisenden in Großbritannien um 1800 zu tadeln für nötig befanden ${ }^{16}$ und der auf englische Traditionen zurückgreifen konnte. Die Resultate waren ausgezeichnet. Wir erinnern hier nur an Withering (Digitalis), Pringle (Militärmedizin), Jenner (Kuhpockenimpfung), Heberden (Angina pectoris), James Lind (Zitrus gegen Skorbut) oder Parkinson. Diese Kliniker waren Empiriker, sprachen aber keine prinzipielle Verurteilung von Chemie und Experiment aus. Auch nicht die hervorragenden Chirurgen wie Percival Pott, John Hunter, John Bell, Astley Cooper, Benjamin Brodie, die, wie die Pariser Chirurgen, Pioniere der pathologischen Anatomie wurden. Es ist bekannt, daß sich Chirurgen wie John Hunter, Charles Bell, Herbert Mayo, Robert Knox und Benjamin Brodie sogar als Experimentalphysiologen und -pathologen betätigten ${ }^{17}$. Paul Cranefield hat erst kürzlich wieder auf einige dieser britischen Forscher-Chirurgen hingewiesen ${ }^{18}$. 
Es muß allerdings festgestellt werden, daß die guten Kliniker eine kleine Minderheit unter den britischen Ärzten darstellten, eine Minderheit, welche entweder als Chirurgen oder in Holland oder in Edinburg (nur die schottischen Universitäten ließen «Dissenters» zu) ausgebildet war. Es ist charakteristisch, daß fast alle britischen Klassiker der Kardiologie (Allan Burns, Joseph Hodgson, James Wardrop, O'Bryen Bellingham, Robert Adams) Chirurgen waren ${ }^{19}$ ! Was die Ärzte betrifft, so konnten seit 1556 nur Cambridge- oder Oxford-Doktoren Fellows des Royal College of Physicians, der zentralen Organisation der inneren Medizin in Großbritannien, werden. Diese Universitäten züchteten aber vor allem Klassizisten und «Gentlemen». Allbutt berichtet, daß 1864 diskutiert werden mußte, ob sich Internisten zu solchen erniedrigenden chirurgischen Eingriffen wie intravenöse Injektion oder Rektaluntersuchungen herablassen sollten ${ }^{20}$. (Kein Wunder, daß die englischen Chirurgen es vorzogen, «Mr.» und nicht «Doctor» genannt zu werden.) Ferner muß gesagt werden, daß die Massen weder von Chirurgen noch von den klassizistischen Doktoren noch von den hervorragenden Empirikern behandelt wurden, sondern von den Apothekern, die in dieser Zeit die praktischen Ärzte Großbritanniens waren, d.h. dieselbe Rolle spielten wie die Wundärzte in Deutschland oder die Officiers de santé in Frankreich. Es war darum auch z. B. möglich, daß der bekannteste britische Psychiater um 1800, John Haslam, Apotheker war!

Es gab unter den "physicians» große Naturforscher wie Thomas Young (Wellentheorie des Lichts), Charles Wells (Eiweiß im Urin nach Scharlach, natürliche Auslese) oder William Hyde Wollaston (untersuchte Harnsteine und gichtige Harnsäuredepots vor William Prout, entdeckte Rhodium und Palladium); aber ihre naturwissenschaftlichen Forschungen spielten keine Rolle für ihre Praxis ${ }^{21}$.

In Anbetracht des empirischen Charakters der britischen Medizin ist es kein Wunder, daß sie die Pariser Spitalmedizin leicht übernahm und mit Robert James Graves und William Stokes in Dublin, mit Richard Bright, Thomas Hodgkin und Thomas Addison im Guys-Spital in London Gleichwertiges leistete.

Das ist also die keineswegs einheitliche Situation um 1800: In Großbritannien ein Gemisch von Klassizisten, Empirikern und wissenschaftlichen Chirurgen. In Frankreich eine von wissenschaftlichem Geist getragene klinische Medizin, welche aber die Naturwissenschaften vernachlässigt und in eine Sackgasse gerät. In Deutschland eine von unwissenschaftlichem Geist getragene Medizin, die eigentlich schon in einer Sackgasse anfängt und darum um 1850 einen großen Umschwung erlebt und die Labormedizin schafft, welche schließlich die ganze Welt 
erobert. Stehen wir vielleicht gegenwärtig am Ende dieser Labormedizin, welche durch eine Kombination von «Büromedizin», Schamanismus, Computern und Barfußärzten abgelöst werden soll?

Anmerkungen

${ }^{1}$ Ackerknecht, Erwin H., Elisha Bartlett and the philosophy of the Paris School. Bull. Hist. Med.24 (1950) $49 \mathrm{f}$.

2 Daremberg, Charles, Histoire des sciences médicales, Paris 1870, Bd. II, S. 1201.

${ }^{3}$ Ackerknecht, Erwin H., Medicine at the Paris Hospital 1794-1848, Baltimore 1967, S. 4. Meine Ausführungen zur französischen Situation sind in diesem Buch belegt.

4 Alibert, Jean-Louis, Eloges historiques, Paris 1806, S. 370.

5 Trousseau, Armand, Clinique médicale, 3 Bde., Paris 1873, Bd. I, S. 139.

${ }^{6}$ Schelling in Jahrbuch der Medizin als Wissenschaft, Bd. I, Tübingen 1806, S. $1 \mathrm{ff}$. Die «Romantische Medizin» ist zusammenfassend vor allen Dingen von Ernst Hirschfeld, Kyklos 3 (Leipzig 1930) 1-89, und Werner Leibbrand (Hamburg/ Leipzig 1937) behandelt.

${ }^{7}$ Schelling, Friedrich Wilhelm, Sämtliche Werke, 14 Bände, Stuttgart 1856, Bd. 2, S. 70.

${ }^{8}$ Hirschfeld, l.c. S. 12 ff.

${ }^{9}$ Schelling, Jahrbuch, Bd. I, 2, S. $1 \mathrm{ff}$.

${ }^{10}$ Görres, Joseph, Exposition der Physiologie, Koblenz 1805, S. 2.

${ }^{11}$ Pixberg, H., Novalis als Naturphilosoph, Gütersloh 1928.

12 Hirschfeld, l.c. S. 34 .

${ }^{13}$ Hirsch, August, Geschichte der Medicinischen Wissenschaften in Deutschland, München 1893, S. 718-720.

${ }^{14}$ Hufeland, Christoph Wilhelm, Rückblick auf mein Leben. Jahrbuch der praktischen Arzneikunde 76 (1833) $1 \mathrm{ff}$.

${ }^{15}$ Lesky, Erna, Die Wiener Medizinische Schule im 19.Jahrhundert, Graz/Köln 1965.

${ }^{16}$ Buchholz, E., Großbritannische Reiseeindrücke deutscher Ärzte, Frankfurt 1960, S. 56.

17 Parker, G., The early history of surgery in Great Britain, London 1920, S. 135-192.

18 Cranefield, Paul, The way in and the way out, New York 1974; id. Introduction to H.Mayo, Anatomical Commentaries, New York 1975; id. Introduction to A. Walker, Documents and Dates, New York 1973.

19 Bedford, D. E., The surgeon-cardiologists. Brit. Heart J. 29 (1967) 461-468.

20 Allbutt, Thomas Clifford, The historical relations of medicine and surgery, London 1905, S. 1.

${ }^{21}$ Chaplin, Arnold, Medicine in England during the reign of George III, London 1919, S. 51-53, 56-65. 


\section{Summary}

The relations between clinical medicine and science around 1800 were different in France, Germany, and Great Britain. In France clinical medicine took a new rise through mere observation in the large hospitals and autopsies. Under the negative impressions of the premature application of physics and chemistry during the $17^{\text {th }}$ and of the systems of the $18^{\text {th }}$ century she used science at best as "ancillary sciences". She had no use for experimental physiology, microscopy, and biochemistry. This lead her into a dead end.

In Germany clinical medicine became completely subservient to the romantic "Naturphilosophie» of Schelling and Cy., which she erroneously called science. She became internationally leading around 1850 when she abandoned speculation for actual experimental science.

In Great Britain a certain elite in clinical medicine reacted against iatrophysics and iatrochemistry through an empirical attitude without condemning science. Certain surgeons were even outstanding experimentalists. The majority of University graduates remained "classicists". The majority of the population was treated by apothecaries.

Prof. Dr. med. Erwin H. Ackerknecht

Ottikerstraße 42

8006 Zürich 